\title{
Common Space Analysis of Several Versions of The Wechsler Intelligence Scale For Children
}

\author{
Richard C. Bell \\ University of Western Australia
}

A joint analysis was made of three versions of the Wechsler Intelligence Scale for Children using an individual differences multidimensional scaling approach. The versions of the test considered were the original version, an Australian partial revision, and the current revised version. Common Space Analysis of the correlation data from the manuals showed a common three-dimensional structure. There was a compact group of verbal subtests and a more loosely defined group of performance tests. Arithmetic, Digit Span, and Coding were found to be more peripheral to the battery. No systematic variations in structure could be related to the different age groups.

Three versions of the Wechsler Intelligence Scale for Children have overlapped in use in Australia-the original version (WISCO; Wechsler, 1949), an Australian partial revision (WISCA; Radcliffe \& Trainer, 1968), and a complete revision (WISCR; Wechsler, 1974). An assumption underlying the use of any of these three versions has been that the various levels of the test measure common constructs of "intelligence." From the correlation matrices provided in the manuals, it has been possible through factor analysis to estimate the structure of intelligence as measured by the test for each level. Gault (1954) provided one of the earliest analyses of the WISCO; and subsequent analyses confirmed these findings, as summarized in reviews by Littell (1960) and Zimmerman and Woo-Sam (1972).

A problem of these analyses has been the relating of factor structures from one age group to another. For the analyses of the WISCO, the problem was not outstanding in that there were only three sets of data to consider; however, the WISCR manual presented 12 correlation matrices. Kaufmann (1975) showed the problems inherent in a simple approach to these data, and later analyses by Silverstein (1977) attempted to overcome this problem by the use of congruence coefficients (although there was then the problem of comparing congruence coefficients). Conger, Conger, Farrell, and Ward (1979) also faced the problem of interpreting congruence coefficients in their analysis of WISCR, based on profile reliabilities.

The assumption that all age groups have an identical factor structure is perhaps rather strong. A more realistic approach might allow for some systematic changes in structure by age. The approaches outlined above do not facilitate these kind of analyses. Either factor analysis across groups (Jöreskog, 1971) or individual differences multidimensional scaling is more appropriate.

APPLIED PSYCHOLOGICAL MEASUREMENT

Vol. 5, No. 1, Winter 1981. pp. 125-132

(C) Copyright 1981 Applied Psychological Measurement Inc. 
Although differences between age group structures might be reasonable within a particular form of the WISC, it might be expected that similar structures would be obtained for similar age groups across forms. Rowe (1976) used canonical variate analysis to assess the similarity of WISCO and WISCR. Canonical variate analysis, however, is a statistical rather than a psychometric technique and is not amenable to construct interpretations (Cliff \& Krus, 1976) although positive statements may be made about the amount of common variance.

An individual differences multidimensional scaling model enables comparisons to be made of both between-age and between-form differences. The fitting of such a model to the WISC data involved an assumption that the correlation matrices were gramian (i.e., scalar product matrices). Comparisons of results with those obtained from factor analysis would not be simple, since the latter are "scale-free," enabling either correlation or covariance matrices to be analyzed. Furthermore, factor analytic interpretations are based on dimensional salience; whereas in multidimensional space, with the origin located at the centroid, interpretation is usually based on the configuration of points. For comparisons to be made, one solution would have to be "transformed" to similarity with the other, as indicated by Schonemann and Carroll (1970).

The particular approach adopted here was the Common Space Analysis (COSPA) of Schonemann (1972; Schonemann, Carter, \& James, 1976, 1977; Schonemann, James, \& Carter, 1978), which enables the fitting of a model consisting of a common structure and a series of weights for each age group. The model is that of Horan (1969), which is similar to the well known INDSCAL model of Carroll and Chang (1970), although Horan's model has the advantage of facilitating tests of fit.

\section{Method}

\section{The Model}

Most individual difference multidimensional scaling models have a similar basis in that the scaled distance between stimuli $i$ and $j$ for person $k$ can be represented by a distance function

$$
d_{i j k}^{2}=\left(a_{i}-a_{j}\right) w_{k}^{2}\left(a_{i}-a_{j}\right), i, j=1, p ; k=1, N
$$

where $a$ gives coordinates in the common space and $W_{k}^{2}$ is a positive definite diagonal matrix of subject weights.

In scalar product terms

$$
\mathrm{B}_{\mathrm{k}}=\mathrm{A} \mathrm{w}_{\mathrm{k}}^{2} \mathrm{~A}^{\prime}
$$

where $B_{k}$ is the $p \times p$ scalar products matrix derived from the distances of the $k^{\text {th }}$ person and $A$, the $p \times$ $m$ ( $m$-dimensional) matrix of $p$ stimulus vectors.

This is Horan's model. The model of Carroll and Chang (1970) is similar but with an additional constraint in that all $B_{k}$ are normalized to have the same variance. The advantage of COSPA (or ALSCAL; Takane, Young, \& de Leeuw, 1977) is that comparisons may be made among the subject weights, since the joint normalization gives an unconditional solution (MacCallum, 1978).

The model of Equation 2 can be broken down into two tests of fit. The first hypothesis is that the $B_{k}$ for all $N$ persons are in the same $m$-dimensional column space, which can be tested by measuring the proportion of variance in the scalar product matrix $B_{k}$ that can be accounted for by the common space assumption model. Schonemann et al. (1978) proposed the index

$$
\mathrm{V}_{\mathrm{k}}=\operatorname{Var}\left(\mathrm{A}^{*} \mathrm{C}_{\mathrm{k}} \mathrm{A}^{* \prime}\right) / \operatorname{Var}\left(\mathrm{B}_{\mathrm{k}}\right), \mathrm{k}=1, \mathrm{~N}
$$

which can be evaluated by recourse to monte carlo data (Schonemann, Carter, \& James, 1977). $C_{k}^{*}$ is the metric of the $\boldsymbol{k}^{\text {th }}$ subject and $\boldsymbol{A}^{*}$ is the common space coordinate matrix. 
The second hypothesis relates to the uniqueness of the solution. If the estimated metric matrix $C_{k}^{*}$ is not diagonal, then the solution is only determined up to any arbitrary nonsingular transformation.

Schonemann et al. (1978) have proposed an index,

$$
\delta_{\mathrm{k}}=\sqrt{\operatorname{tr}\left(\mathrm{R}_{\mathrm{k}}-\mathrm{I}\right)^{2}} \overline{\operatorname{m(m-1)}}, \mathrm{k}=1, \mathrm{~N}
$$

where $R_{k}$ is obtained by normalizing the matrix $T^{\prime} C_{k}^{*} T$ by columns and rows, $T$ being an optimal rotation.

If both assumptions are met, $V_{k}$ should be close to 1.0 , and $\delta_{k}$ close to 0.0 . The index $V_{k}$ has been noted (Schonemann et al., 1978) as being similar to Carroll and Chang's (1970) correlational index of fit.

\section{Analysis}

Using the correlation matrices from the various manuals, as indicated in Table 1, Horan's (1969) model was fitted to the data using COSPA (Schonemann et al., 1978).

Table 1

Fit of Horan's Individual Difference Multidimensional Scaling Model to WISC Data

\begin{tabular}{ccccccc}
\hline & \multicolumn{7}{c}{ Dimension } \\
\cline { 2 - 7 } WISCO & $\mathrm{V}$ & $\delta$ & $\mathrm{V}$ & $\delta$ & $\mathrm{V}$ & $\delta$ \\
$7 \frac{1}{2}$ & .37 & .04 & .48 & .08 & .57 & .10 \\
$10 \frac{1}{2}$ & .43 & .01 & .57 & .05 & .63 & .05 \\
$13 \frac{1}{2}$ & .44 & .09 & .57 & .13 & .67 & .09 \\
WISCA & & & & & & \\
8 & .50 & .05 & .61 & .06 & .70 & .06 \\
10 & .48 & .16 & .58 & .09 & .67 & .06 \\
12 & .47 & .13 & .58 & .10 & .70 & .06 \\
WISCR & & & & & & \\
$6 \frac{1}{2}$ & .46 & .14 & .60 & .09 & .69 & .05 \\
$7 \frac{1}{2}$ & .59 & .06 & .66 & .10 & .74 & .08 \\
$8 \frac{1}{2}$ & .50 & .01 & .61 & .06 & .69 & .06 \\
$9 \frac{1}{2}$ & .50 & .02 & .60 & .02 & .67 & .04 \\
$10 \frac{1}{2}$ & .47 & .05 & .60 & .04 & .69 & .05 \\
$11 \frac{1}{2}$ & .50 & .04 & .61 & .03 & .70 & .02 \\
$12 \frac{1}{2}$ & .40 & .11 & .48 & .11 & .56 & .07 \\
$13 \frac{1}{2}$ & .45 & .07 & .57 & .07 & .63 & .06 \\
$14 \frac{1}{2}$ & .45 & .16 & .57 & .08 & .72 & .13 \\
$15 \frac{1}{2}$ & .39 & .12 & .48 & .08 & .58 & .07 \\
$16 \frac{1}{2}$ & .48 & .07 & .56 & .07 & .62 & .08 \\
Total & .44 & .07 & .52 & .07 & .60 & .05 \\
Average & .46 & .08 & .57 & .07 & .66 & .07 \\
V crit & .30 & & .41 & & .54 & \\
$\delta$ crit & & .03 & & .09 & & .13 \\
crit & & .09 &
\end{tabular}




\section{Results}

The test indices $V_{k}$ and $\delta_{k}$ for solutions in two, three, and four dimensions together with levels that distinguish true from random data (from Schonemann et al., 1977) are shown in Table 1. The levels were based on upper deciles for $V_{k}$ and lower deciles for $\delta_{k}$. It can be seen that for all three solutions the common space assumptions were met (i.e., $V_{k}>V_{c r i t}$ ). For the diagonality conditions (i.e., $\delta_{k}<$ $\left.\delta_{c r i t}\right)$, in two dimensions 15 matrices violated the assumptions, in three dimensions 4 matrices violated the assumptions, and all matrices were satisfactory for four dimensions.

The three-dimensional solution was chosen for consideration, as the violations were not substantial, although caution would be needed in interpreting results for WISCO at $13 \frac{1}{2}$ years. Investigation of the weights for these groups in a four-dimensional solution did not reveal any consistent difference in pattern from the other groups. The coordinates for the WISC subtests in three-dimensional space are shown in Table 2. To facilitate interpretation of these data, a clustering of the Euclidean distances between WISC subtests in the COSPA 3 space was undertaken using Johnson's (1967) complete linkage hierarchical clustering (the "diameter" method). Results are shown in Figure 1. The weights for the 18 sources of data are shown in Table 3.

Correlations were computed between age levels and the three weights; and coefficients of $.36, .08$, and .02 , respectively, were obtained, none of which were significant $(p>.05)$. Differences between the weights for three forms were assessed by analyses of variance, and no significant differences were found $(p>.05)$ among weights for WISCO, WISCA, and WISCR.

\section{Discussion}

Previous analyses of the WISCO and WISCR data have used factor analysis of the separate correlation matrices and, in doing so, have posited several structures for the WISC. The most basic distinction has been between two- and three-factor solutions. Silverstein (1977) obtained both two- and three-factor solutions, with a two-factor solution being more similar (in terms of high congruence coefficients) for the different age groups. Kaufman (1975) obtained two-factor solutions for six age

Table 2

\begin{tabular}{lrrr} 
COSPA 3-Dimensional Solution for WISC Subtests \\
\hline \multirow{2}{*}{ WISC Subtest } & \multicolumn{3}{c}{ Dimension } \\
\cline { 2 - 4 } Information & 1 & 2 & 3 \\
Similarities & .33 & -.15 & .07 \\
Arithmetic & .34 & -.10 & .01 \\
Vocabulary & .00 & -.38 & .04 \\
Comprehension & .38 & -.14 & .09 \\
Digit Span & .41 & -.01 & .12 \\
Picture Completion & -.37 & -.67 & -.12 \\
Picture Arrangement & .05 & .23 & -.33 \\
Block Design & .02 & .31 & .11 \\
Object Assembly & -.13 & .22 & -.21 \\
Coding & -.16 & .40 & -.25 \\
Mazes & -.43 & .07 & .70 \\
& -.43 & .23 & -.23
\end{tabular}




\section{Figure 1}

\section{Complete Linkage Hierarchical Clustering of COSPA Distances Among WISC Subtests}

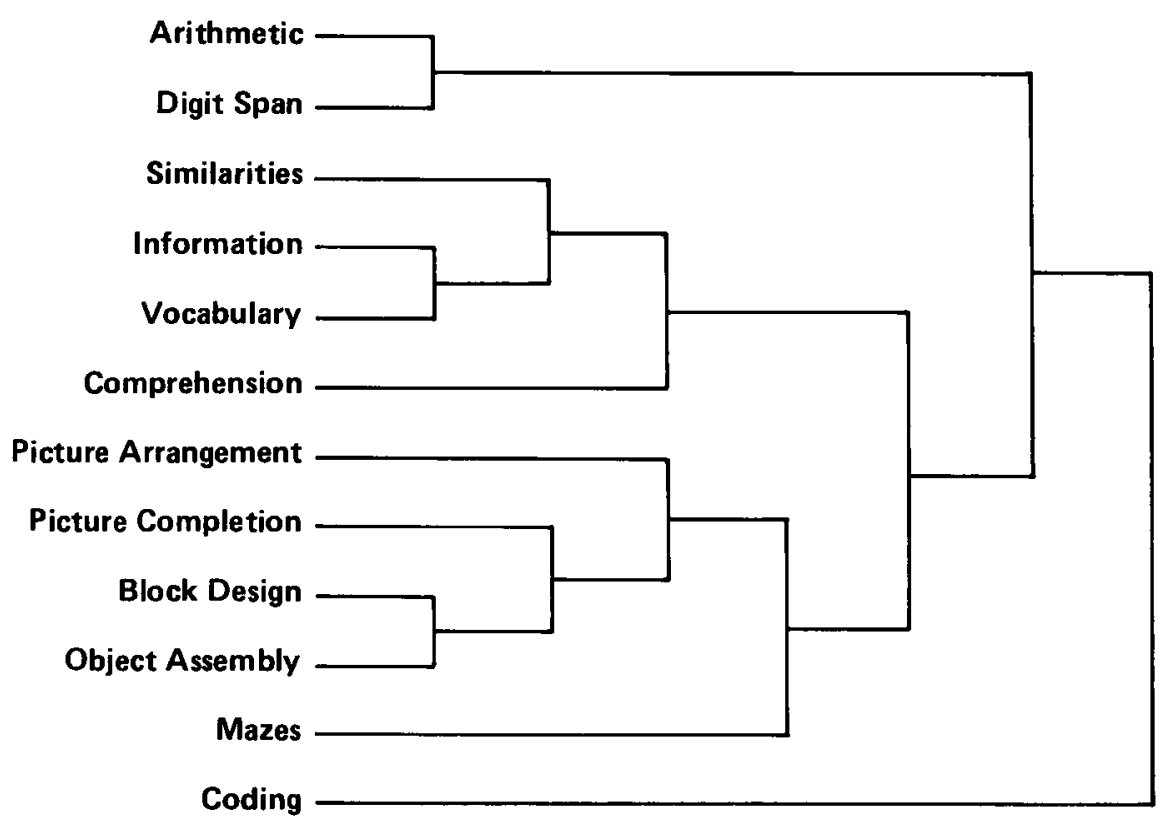

levels and three-factor solutions for the other five. The present analysis indicated that three dimensions (akin to three factors) were important in all, although the variation in age/form weights was least for the third dimension.

Previous studies, as above, identified the first two factors as Verbal and Performance and the third as Freedom from Distractibility. The appropriate verbal and performance subtests would load on the first two; and Coding Mazes, Digit Span, and Arithmetic would load for various matrices on the Freedom from Distractibility factor.

In multidimensional scaling, attempts are usually made to interpret the configuration of points (representing the subtests) rather than the dimensions. The dimensions in this analysis were largely bipolar. The first dimension contrasted the truly verbal subtests (i.e., not including Arithmetic or Digit Span) with the nonverbal active performance tests and Digit Span. The second dimension showed the classic WISC verbal/performance subscale distinction, and the third was dominated by Coding.

When the configuration of the subtests was considered as in the hierarchical clustering, a compact core of verbal subtests-Similarities, Information, Vocabulary, and Comprehension-was evident. Together with a less closely related group of performance tests-Picture Arrangement, Picture Completion, Block Design, Object Assembly, and Mazes-this formed a general core for the battery. Arithmetic, Digit Span, and Coding were less related to the major part of the battery

The isolation of Digit Span and Coding was expected; but the findings for Arithmetic were something of a surprise, although both Kaufman (1975) and Silverstein $(1977,1980)$ reported similar findings. An examination of arithmetic items showed them to be similar to subtests in the Kit of Cognitive Reference Tests (French, Ekstrom, \& Price, 1963), which purport to measure general reasoning. The 
Table 3

COSPA Weights for the WISC Data Sources

\begin{tabular}{crrr}
\hline Form and & \multicolumn{3}{c}{ Weights } \\
\cline { 2 - 4 } age & \multicolumn{1}{c}{ 1 } & \multicolumn{1}{c}{3} \\
\hline WISCO & & & \\
$7 \frac{1}{2}$ & .78 & .88 & .91 \\
$10 \frac{1}{2}$ & .90 & .94 & 1.13 \\
$13 \frac{1}{2}$ & 1.11 & .98 & .97 \\
WISCA & & & \\
8 & .90 & 1.01 & 1.00 \\
10 & 1.09 & .97 & 1.03 \\
12 & .93 & .96 & 1.10 \\
WISCR & & & \\
$6 \frac{1}{2}$ & .94 & .94 & 1.02 \\
$7 \frac{1}{2}$ & 1.18 & 1.07 & .92 \\
$8 \frac{1}{2}$ & 1.02 & .96 & 1.04 \\
$9 \frac{1}{2}$ & 1.05 & 1.09 & 1.00 \\
$10 \frac{1}{2}$ & .85 & 1.15 & 1.07 \\
$11 \frac{1}{2}$ & .99 & 1.00 & 1.01 \\
$12 \frac{1}{2}$ & .90 & .89 & .98 \\
$13 \frac{1}{2}$ & .89 & .81 & 1.09 \\
$14 \frac{1}{2}$ & .93 & 1.10 & 1.02 \\
$15 \frac{1}{2}$ & 1.06 & 1.27 & .94 \\
$16 \frac{1}{2}$ & 1.36 & .86 & .95 \\
Tota1 & 1.11 & 1.12 & .82 \\
Mean & 1.00 & 1.00 & 1.01 \\
SD & .13 & .12 & .07 \\
\hline
\end{tabular}

isolation of WISC arithmetic in the configuration would suggest that this test does not measure general reasoning.

With respect to the different emphases on the dimensions of the subtest space, there were no differences between the three forms of the WISC. Similarly, there were no systematic trends by age-at least as far as linear relationships were considered, although inspection of Table 3 suggests that there are no relationships of any form between age and dimension weights. Dimension 1 was weighted most substantially by WISC at $161 / 2$ years and least by WISCO at $71 / 2$ years; Dimension 2 was weighted most by WISCR at 151/2 years and least by WISCR at 131/2 years; Dimension 3 was weighted most by WISCO at $10^{1 / 2}$ years and least by the WISCR total data. The WISCR total data did not fall close to a central position in the weights, and WISCR at $11^{1} \frac{1}{2}$ years was, in fact, the most central data.

\section{Conclusions}

It was concluded that there was a common three-dimensional structure to the WISC data from the three forms of the WISC considered. The structure was a more refined structure than Wechsler's simple verbal/performance dichotomy, and several subtests could be considered less central to the 
battery. There were no significant differences between the forms of the WISC nor were there relationships between age level and structure, indicating that intelligence, as measured by the different sections of the different forms of the WISC, is essentially the same in structure.

The results from the individual differences multidimensional scaling were substantially in agreement with results from factor analytic and clustering studies, pointing to a usefulness in adopting such approaches to the analysis of WISC and other similar test data, either through the present approach in COSPA or the more extensive possibilities in ALSCAL.

\section{References}

Carroll, J. D., \& Chang, J. J. Analysis of individual differences in multidimensional scaling via an $N$ way generalization of "Eckart-Young" decomposition. Psychometrika, 1970, 35, 283-319.

Cliff, N., \& Krus, D. J. Interpretation of canonical analysis: Rotated vs. unrotated solutions. Psychometrika, 1976, 41, 35-42.

Conger, A. J., Conger, J. C., Farrell, A. D., \& Ward, D. What can WISC-R measure? Applied Psychological Measurement, 1979, 3, 421-436.

French, J. W., Ekstrom, R. B., \& Price, L. A. Manual for kit of reference tests for cognitive factors (rev. ed.). Princeton, NJ: Educational Testing Service, 1963.

Gault, U. Factorial patterns on the Wechsler Intelligence Scales. Australian Journal of Psychology, 1954, 6, 85-90.

Horan, C. B. Multidimensional scaling: Combining observations when individuals have different perceptual structures. Psychometrika, 1969, 34, 139-165.

Johnson, S. C. Hierarchical clustering schemes. Psychometrika, 1967, 32, 241-254.

Jöreskog, J. G. Simultaneous factor analysis in several populations. Psychometrika, 1971, 36, 409-426.

Kaufman, A. S. Factor analysis of the WISC-R at 11 age levels between 6 and 16 years. Journal of Consulting and Clinical Psychology, 1975, 43, 135-147.

Littell, W. M. The WISC-a review of a decade of research. Psychological Bulletin, 1960, 57, 132-162.

MacCallum, R. C. Effects of conditionality on INDSCAL and ALSCAL weights. Psychometrika, 1977, 42, 297-305.

Radcliffe, J. A., \& Trainer, F. E. Manual for the Australian revision of the Wechsler Intelligence Scale for Children. Melbourne: Melbourne Council for Educational Research, 1968.

Rowe, H. A. H. The comparability of WISC and WISCR (Occasional Paper No. 10). Hawthorne, Victoria, Australia: Australian Council for Educational Research, 1976.
Schonemann, P. H. An algebraic solution for a class of subjective metrics models. Psychometrika, 1972, 37, 441-451.

Schonemann, P. H., \& Carroll, R. M. Fitting one matrix to another under choice of a central dilation and a rigid motion. Psychometrika, 1970, 35, 245-256.

Schonemann, P. H., Carter, F. S., \& James, W. L. Contributions to subjective metrics scaling: $I$. COSPA, a fast method for fitting and testing Horan's model, and an empirical comparison with INDSCAL and ALSCAL (Institute Paper No. 587). West Lafayette, IN: Purdue University, Krannert Graduate School, 1976.

Schonemann, P. H., Carter, F. S., \& James, W. L. Contributions to subjective metrics scaling: II. A statistical test and approximate norms for evaluating the fit of Horan's model with COSPA (Institute Paper No. 623). West Lafayette, IN: Purdue University, Krannert Graduate School, 1977.

Schonemann, P. H., James, W. L., \& Carter, F. S. COSPA: Common space analysis-A program for fitting and testing Horan's subjective metrics model. Journal of Marketing Research, 1978, 15, 268-272.

Silverstein, A. B. Alternative factor analytic solutions for the Wechsler Intelligence Scale for Children-Revised. Educational and Psychological Measurement, 1977, 37, 121-124.

Silverstein, A. B. Cluster analysis of the Wechsler Intelligence Scale for Children-Revised. Educational and Psychological Measurement, 1980, 40, 51-54.

Takane, Y., Young, F. W., \& de Leeuw, J. Nonmetric individual differences in multidimensional scaling: An alternating least squares method with optimal scaling features. Psychometrika, 1977, 42, 7-67.

Wechsler, D. WISC Manual. New York: Psychological Corporation, 1949.

Wechsler, D. WISC-R Manual. New York: Psychological Corporation, 1974. 
Zimmerman, I. L., \& Woo-Sam, J. Research with the Wechsler Intelligence Scale for Children: 1960-1970. Psychology in the Schools, 1972, 9, 232-271.

\section{Author's Address}

Send requests for reprints or further information to Richard C. Bell, Research Unit in University Education, University of Western Australia, Nedlands, Western Australia, 6009, Australia. 